\title{
Relative Toxicity of Certain Pesticides to Lagenidium giganteum (Oomycetes: Lagenidiales), a Fungal Pathogen of Mosquito Larvae ${ }^{1}$
}

\author{
T. L. MERRIAM AND R. C. AXTELL \\ North Carolina State University, Raleigh, North Carolina 27650
}

\begin{abstract}
Environ. Entomol. 12: 515-521 (1983)
ABSTRACT Laboratory experiments were conducted to determine the relative toxicity of certain insecticides, herbicides, and fungicides to the vegetative growth and zoospore production in North Carolina (NC) and Louisiana (LA) isolates of Lagenidium giganteum. Zoospore production in both isolates was less tolerant than mycelial growth to most of the pesticides tested and, thus, the $\mathrm{IC}_{50}$ values (inhibition concentration) for mycelial growth rate on agar underestimated toxicity. The most toxic pesticides to one or both isolates of $L$. giganteum were captan, gamma-BHC. DDT, toxaphene, chlorpyrifos, and fenthion. These pesticides produced at least $10 \%$ inhibition of mycelial growth rate or completely inhibited zoospore production at concentrations below 5 parts per million (ppm). The least toxic pesticides caused a $10 \%$ inhibition in mycelial growth rate and failed to inhibit zoospore production at concentrations greater that $50 \mathrm{ppm}$. These were diflubenzuron, permethrin, temephos, and propoxur (LA isolate only). Pesticides of intermediate toxicity were: malathion, carbaryl, methoprene, alachlor, and atrazine. The two isolates differed substantially in their tolerance of the following pesticides: malathion, chlorpyrifos, toxaphene, carbaryl, propoxur, permethrin, and methoprene. At their recommended rates of application for control of mosquito larvae, methoprene, fenthion, malathion, and temephos would probably be compatible with both isolates of L. giganteum.
\end{abstract}

The fungus Lagenidium giganteum Couch (Oomycetes: Lagenidiales) is a promising candidate as a biological control agent for mosquitoes (Federici 1981). Although originally described as a weak, facultative parasite of mosquito larvae, Daphnia, and copepods (Couch 1935), L. giganteum has been shown to be virulent pathogen of several genera of culicid larvae (McCray et al. $1973 \mathrm{ab}$ ). The fungus develops rapidly and kills host mosquito larvae usually within $72 \mathrm{~h}$, can easily be produced in vitro, is apparently safe to nontarget organisms, and is persistent because it has the ability to overwinter in nature (Glenn and Chapman 1978). In preliminary field tests, L. giganteum gave variable control of Culex tarsalis (Coquillett) larvae in rice fields and drainage ditches (McCray et al. 1973b),

Although $L$. giganteum has received much study during the past decade, little is known about its tolerance to environmental factors. In laboratory and field studies, Jaronski and Axtell (1982) found that zoosporogenesis by $L$. giganteum was inhibited in water with low to moderate levels of organic pollution. Merriam and Axtell (1982) examined the salinity tolerance of two isolates of $L$. giganteum and found that infection in Aedes taeniorhynchus (Wiedemann) larvae by each isolate was reduced by $50 \%$ in water containing only ca. 0.5 parts per thousand $\mathrm{NaCl}$, as compared with the percent infection achieved in distilled water.

Researchers have reported that entomogenous fungi are adversely affected by certain pesticides, including insecticides and fungicides (Ramaraje Urs et al. 1967, Olmert and Kenneth 1974). Comprehensive reviews on the compatibility of pesticides with entomogenous fungi have been written by Roberts and Campbell (1977), and Jacques and Morris (1981). Data are lacking, however, on the effects of pesticides on $L$. giganteum. Due to the

Paper No. 8383 of the Journal Series of the N.C. Agric. Res. Serv. Received for publication I July 1982 use of certain insecticides as larvicides for mosquito control and herbicides for weed control, and the contamination of aquatic habitats by pesticide transport from agricultural lands, the compatibility of $L$. giganteum with various pesticides in the aquatic environment may be a critical factor in its ability to survive and function as an effective microbial control agent for mosquitoes. Therefore, the purpose of this study was to determine the relative toxicity of selected pesticides to two isolates of L. giganteum.

\section{Materials and Methods}

Sources and Maintenance of Fungi

The two isolates of $L$. giganteum used in this study were: (1) North Carolina strain (NC), isolated by A. L. Knight from infected mosquito larvae in Chapel Hill, N.C., in 1979; and (2) Louisiana strain (LA), isolated by Glenn and Chapman (1978) from infected Culex territans (Walker) in a blackgum swamp near Moss Bluff, La. Stock cultures of both isolates were routinely maintained on Peptone-Yeast Extract-Glucose (PYG) agar $(0.125 \%$ peptone, $0.125 \%$ yeast extract, $0.3 \%$ glucose, and $2.0 \%$ agar).

\section{Pesticides}

Pesticides included in this study were selected as representative examples of chemicals which might occur in aquatic systems as a result of larviciding for mosquito control or via transport from agricultural lands. Experiments were conducted to study the effects of the following pesticides on the mycelial growth of L. giganteum. (1) Organophosphate insecticides: chlorpyrifos [diethy] $O$-(3,5,6-trichloro-2-pyridyl) phosphorothioate]; fenthion ( $O, O$-dimethyl $O$-(4-methylthiam-tolyl) phosphorothioate]; malathion [diethyl mercaptosuccinate $\mathrm{S}$-ester with $O, O$-dimethyl phosphorodithioate] and temephos $\left[O, O^{\prime}\right.$-(thiodi-p-phenylene) $O^{\prime}, O^{\prime}, O^{\prime}, O^{\prime}$, tetra 
methyl phosphorothioate]. (2) Carbamate insecticides: carbaryl [1-naphthyl methylcarbamate] and propoxur [0isopropoxyphenyl methylcarbamate]. (3) Organochlorine insecticides: gamma-BHC $[1,2,3,4,5,6$-hexachlorocyclohexane, gamma isomer]; DDT [1,1,1-trichloro2,2-bis( $p$-chlorophenyl) ethane] and toxaphene [chlorinated camphene containing 67 to $69 \%$ chlorine]. (4) Insect growth regulators: diflubenzuron [1-(4-chlorophenyl)-3-(2,6-diflurobenzoyl) urea] and methoprene [isopropyl (E,E)-11-methoxy-3,7,11-trimethyl-2,4-dodecadienoate]. (5) Synthetic pyrethroid insecticide: permethrin $[m$-phenoxybenzyl $( \pm)$-cis, trans-3- $(2,2-$ dichlorovinyl)-2,2-dimethyl-cyclopropanecar

boxylate]. (6) Herbicides: alachlor [2-chloro-2', 6'-diethyl-N-(methoxymethyl)acetanilide] and atrazine (2chloro-4-ethylamino-6-isopropylamino-s-triazine]. (7) Fungicide: captan [N-trichloromethylthio)-4-cyclohexene-1,2-dicarboximide].

The organophosphate and carbamate insecticides, herbicides, and captan were purchased from Chem Service, West Chester, $\mathrm{Pa}$. The organochlorine insecticides were manufactured by City Chemical Corp., New York, N.Y. Diflubenzuron was supplied by Thompson-Haywood Chemical Co., Kansas City, Kans. Methoprene was furnished by Zoecon Corp., Palo Alto, Calif. Permethrin was supplied by Burroughs Wellcome Co., Research Triangle Park, N.C.

\section{Mycelial Growth}

Each isolate of $L$. giganteum was grown in PYG agar in standard petri dishes and incubated at 25 to $27^{\circ} \mathrm{C}$. After 5 days, circular plugs of agar $(5.0 \mathrm{~mm}$ in diameter $)$ bearing the most recent mycelial growth from the outer edge of the culture were made with a sterile, metal corkborer. The agar plugs were transferred, mycelial growth downward, to the center of a series of sterile, 100-mmdiameter petri dishes (one plug per dish) containing PYG agar and a pesticide at its recommended field rate and several concentrations higher and lower. These petri dishes had been prepared by incorporating the pesticide (technical grade dissolved in acetone) at the proper concentration into liquid PYG agar $\left(48\right.$ to $\left.50^{\circ} \mathrm{C}\right)$ to give a final volume of $200 \mathrm{ml}$ per flask. The pesticide-agar mixture was agitated and then poured into 10 petri dishes (ca. $20 \mathrm{ml}$ per dish), 5 dishes per isolate. Final acetone concentrations in the PYG agar were $1 \%$ or less, since preliminary experiments comparing the mycelial growth rate of each isolate on PYG agar with their respective growth rates on PYG agar containing various concentrations of acetone showed $2 \%$ inhibition of mycelial growth rate at 1.4 and $1.2 \%$ acetone for the $\mathrm{NC}$ and LA isolates, respectively. Additional petri dishes containing only PYG agar served as controls for each isolate. The experiment was repeated three times. The surface area of the mycelium growing on the agar in each petri dish was determined at 5 and 7 days postinoculum by using an electronic planimeter. After correcting for the surface area of the agar plug used for inoculating the petri dishes, the radial mycelial growth rate (RMGR) in each petri dish during growth period no. 1 (days 1 to 5) and growth period no. 2 (days 6 and 7) was calculated. A mean RMGR was then calculated for each isolate at each pesticide concentration and control using the corresponding RMGRs from the two growth periods. Data were plotted by using probit transformations of the mean RMGRs (each expressed as the percent inhibition [I] compared with the control) and $\log _{10}$ transformations of the pesticide concentrations. The concentrations of each pesticide for various percent inhibition in mycelial growth rate (e.g., $\mathrm{IC}_{50}, \mathrm{IC}_{10}$ ) and respective $95 \%$ fiducial limits were calculated for each isolate (Barr et al. 1979).

\section{Zoospore Production}

Additional experiments were conducted to examine the effects of the pesticides on the ability of L. giganteum to produce viable zoospores, the infective stage of the fungus. Each isolate was cultured on whole hemp seed extract agar (WHS) by methods described previously by Merriam and Axtell (1982).

Tests were conducted in $250-\mathrm{ml}$ glass beakers containing sterile, distilled water and a pesticide (technical grade dissolved in acetone) to give a final concentration of pesticide in water equal to its mycelial $\mathrm{IC}_{10}$ value (wt/ vol). Total volume was $200 \mathrm{ml}$ per beaker. Because permethrin, toxaphene, methoprene and diflubenzuron were nearly insoluble in water at their respective mycelial $\mathrm{IC}_{10}$ concentrations (and lower), a $0.01 \%$ solution of Tween 80 in sterile, distilled water was used to increase their water solubility. There were three beakers for each pesticide and distilled water control. Three additional beakers containing sterile, distilled water, acetone, and $0.01 \%$ Tween 80 (when used) were included in the tests. Acetone concentrations equal to $2 \%$ or less and a Tween 80 concentration of $0.01 \%$ in distilled water were used, since preliminary tests showed that these concentrations of acetone and Tween 80 did not adversely affect zoospore production and germination by L. gigatenum, including those instances when acetone and Tween 80 were used together.

The WHS agar bearing 7-day-old mycelial growth in several petri dishes was cut into 1.5 -cm-diameter sections with a sterile, metal cork-borer. Three agar sections were immersed, mycelial side upwards, into the liquid in each beaker at random to minimize any intraor inter-dish difference in zoospore production. The beakers were covered to prevent evaporation. The agar sections were removed and examined microscopically for evidence of the production of viable zoospores. The agar sections of each isolate were examined at two different times to determine (1) if the pesticides had inhibited or delayed zoosporogenesis and (2) if the motile zoospores had germinated on the WHS agar sections in the beakers. Because the LA isolate produced zoospores sooner after immersion in distilled water than did the $\mathrm{NC}$ isolate, the LA isolate agar sections were examined at 12 and $18 \mathrm{~h}$ postimmersion, whereas those of the $\mathrm{NC}$ isolate were examined at $18 \mathrm{~h}$ post immersion. The criterion for viable zoospore production was the visual presence of both motile zoospores and germinated zoospores. If viable zoospores were not observed in the treat- 
ment containing each pesticide at its mycelial $\mathrm{IC}_{10}$ concentration, the test was repeated, using an arbitrary series of lower pesticide concentrations based on the dose-effect line for each isolate until viable zoospore production was observed.

\section{Results}

The toxicity of the pesticides to mycelial growth and zoospore production of the NC and LA isolates of $L$. giganteum is shown in Table 1. Chlorpyrifos was the most toxic of the organophosphate insecticides to both isolates. Although the mycelial $\mathrm{IC}_{50}$ and $\mathrm{IC}_{10}$ values for chlorpyrifos were relatively high for both isolates, extensive plasmolysis was observed when the mycelia were placed into distilled water containing chlorpyrifos at $\mathrm{IC}_{10}$ and $\mathrm{IC}_{5}$ concentrations. The LA isolate was more tolerant to chlorpyrifos than was the $\mathrm{NC}$ isolate, since the former had higher $\mathrm{IC}_{50}$ and $\mathrm{IC}_{10}$ values and produced viable zoospores in water containing $1.4 \mathrm{ppm}$ of chlorpyrifos.

Fenthion and malathion showed intermediate toxicity to both isolates of $L$. giganteum. The LA isolate was more tolerant to fenthion than was the NC isolate, since the LA isolate successfully produced zoospores and the $\mathrm{NC}$ isolate produced only twisted discharge tubes in distilled water containing $2.3 \mathrm{ppm}$ of fenthion. The NC isolate appeared more tolerant than the LA isolate of malathion, since the $\mathrm{NC}$ isolate produced zoospores in distilled water containing $12.4 \mathrm{ppm}$, and showed 50\% inhibition of mycelial growth rate at a higher malathion concentration than did the LA isolate.

Temephos was the least toxic organophosphate insecticide to the two isolates of $L$. giganteum. The mycelial $\mathrm{IC}_{50}$ and $\mathrm{IC}_{10}$ were relatively high, and both isolates produced zoospores in distilled water containing 65.8 $\mathrm{ppm}$ of temephos. Although no mycelial plasmolysis was observed at the temephos $\mathrm{IC}_{10}$ of the $\mathrm{NC}$ isolate and the $\mathrm{IC}_{10}, \mathrm{IC}_{5}$, and $\mathrm{IC}_{1}$ of the $\mathrm{LA}$ isolate, neither isolate produced discharge tubes.

Gamma-BHC was the most toxic organochlorine insecticide to the mycelial growth and production of zoospores, whereas toxaphene and DDT, respectively, showed less toxicity to both isolates. In terms of the inhibitions of mycelial growth rate, the $\mathrm{NC}$ isolate was more tolerant of all the organochlorine insecticides than was the LA isolate. In contrast, zoosporogenesis in the $\mathrm{NC}$ isolate was less tolerant than that in the LA isolate to DDT and toxaphene, since the NC isolate was unable to produce viable zoospores in distilled water containing these insecticides at concentrations in which the LA isolate successfully produced zoospores. Gamma-BHC was more toxic to the LA isolate than to the $\mathrm{NC}$ isolate, however, since the NC isolate produced zoospores in distilled water containing a higher concentration of gamma-BHC, and had a higher $\mathrm{IC}_{50}$ value than did the LA isolate.

Although the LA isolate produced viable zoospores at its mycelial $\mathrm{IC}_{10}$ for each organochlorine insecticide,

Table 1.-Toxicity of pesticides to mycelial growth and zoospore production of $\mathrm{NC}$ and LA isolates of $L$. giganteum

\begin{tabular}{|c|c|c|c|c|c|c|}
\hline \multirow{3}{*}{ Pesticide } & \multicolumn{3}{|c|}{$\mathrm{NC}$ isolate } & \multicolumn{3}{|c|}{ LA isolate } \\
\hline & \multicolumn{2}{|l|}{$\begin{array}{l}\text { Mycelial inhibition } \\
(\mathrm{ppm})\end{array}$} & \multirow{2}{*}{$\begin{array}{l}\text { Maximum concn } \\
\text { at which } \\
\text { zoospores produced } \\
(\mathrm{ppm})\end{array}$} & \multicolumn{2}{|l|}{$\begin{array}{l}\text { Mycelial inhibition } \\
(\mathrm{ppm})\end{array}$} & \multirow{2}{*}{$\begin{array}{c}\text { Maximum concn } \\
\text { at which } \\
\text { zoospores produced } \\
(\mathrm{ppm})\end{array}$} \\
\hline & $\mathrm{IC}_{50}$ & $\mathrm{IC}_{10}$ & & $\mathrm{IC}_{501}$ & $\mathrm{IC}_{10}$ & \\
\hline \multicolumn{7}{|c|}{ Organophosphate insecticides } \\
\hline Chlorpyrifos & $569.9(316-1.178)^{4}$ & 6.2 & $0.2\left(=\mathrm{IC}_{1}\right)^{\prime \prime}$ & $1.439 .7(907-2.900)^{\prime \prime}$ & 76.1 & $1.4\left(=0.2 \mathrm{IC}_{1}\right)^{b}$ \\
\hline Fenthion & $120.6(87-172)$ & 8.9 & $1.1\left(=I_{1}\right)$ & 54.3 & 2.3 & $2.3\left(=I_{10}\right)$ \\
\hline Malathion & $130.6(16-733)$ & 20.9 & $12.4\left(=1 C_{5}\right)$ & 32.0 & 5.8 & $5.8 \cdot\left(=\mathrm{IC}_{10}\right)$ \\
\hline Temephos & $4,697.0$ & 163.8 & $65.8\left(=\mathrm{IC}_{5}\right)$ & $1.653 .3(1.288-2.320)$ & 345.3 & $65.8\left(=0.68 I_{1}\right)$ \\
\hline \multicolumn{7}{|c|}{ Chlorinated hydrocarbon insecticides } \\
\hline DDT & $1,862.0$ & 38.7 & $1.6\left(=1 C_{1}\right)$ & 586.7 & 6.2 & $6.2^{\circ}\left(=\mathrm{IC}_{10}\right)$ \\
\hline Gamma-BHC & $2.0(2-13)$ & 1.0 & $0.5\left(=I C_{1}\right)$ & 1.5 & 0.3 & $0.3^{r}\left(=\mathrm{IC}_{10}\right)$ \\
\hline Toxaphene & 90.8 & 6.1 & $0.7\left(=I C_{1}\right)$ & 37.4 & 2.0 & $2.0^{x}\left(=\mathrm{IC}_{10}\right)$ \\
\hline \multicolumn{7}{|l|}{ Carbamate insecticides } \\
\hline Carbaryl & $43.7(35-54)$ & 13.4 & $5.1\left(=I_{1}\right)$ & $39.4(32-49)$ & 12.6 & $12.6^{c}\left(=I C_{10}\right)$ \\
\hline Propoxur & 231.3 & 127.6 & $15.7\left(=0.2 \mathrm{IC}_{1}\right)$ & 207.0 & 58.5 & $58.5^{\circ}\left(=\mathrm{IC}_{10}\right)$ \\
\hline \multicolumn{7}{|c|}{ Synthetic pyrethroid insecticides } \\
\hline \multicolumn{6}{|c|}{ Insect growth regulator insecticides } & $88.6\left(=I C_{5}\right)$ \\
\hline Diflubenzuron & $>2,000.0$ & $-=$ & $>100.0$ & $>2.000 .0$ & - & $>100.0^{\circ}$ \\
\hline Methoprene & $6,862.0$ & 461.3 & $10.2\left(=0.2 \mathrm{IC}_{1}\right)$ & 1.952 .1 & 890.3 & $23.5\left(=0.05\left(\mathrm{IC}_{1}\right)\right.$ \\
\hline \multicolumn{7}{|l|}{ Herbicides } \\
\hline Alachlor & 47.2 & 22.8 & $22.8\left(=\mathrm{IC}_{10}\right)$ & 42.7 & 22.4 & $22.4^{x}\left(=I C_{10}\right)$ \\
\hline \multicolumn{6}{|l|}{ Fungicide } & $15.2^{\prime}\left(=\mathrm{IC}_{10}\right)$ \\
\hline Captan & $64.7(51-81)$ & 16.6 & $0.3\left(=0.05 I C_{1}\right)$ & 44.9 & 7.6 & $0.3\left(=0.15 I_{1}\right)$ \\
\hline
\end{tabular}

"The $95 \%$ fiducial limits for the $\mathrm{IC}_{50}$ values are shown in parentheses. Absence of a value indicates there was too much variation in the data to calculate meaningful $95 \%$ fiducial limits.

${ }^{b}$ Values in parentheses indicate degree of mycelial inhibition at this concentration.

Highest concentration tested. 
Table 2.-Ranking of the relative toxicity of certain pesticides to the North Carolina (NC) and Louisiana (LA) isolates of L. giganteum

\begin{tabular}{lll}
\hline \hline \multicolumn{1}{c}{ Toxicity level $^{a}$} & NC isolate & LA isolate \\
\hline Low $\left(\mathrm{Z}_{\mathrm{IC}_{\mathrm{x}}} \geqslant 50 \mathrm{ppm}\right)$ & & \\
& $\begin{array}{l}\text { Diflubenzuron } \\
\text { Temephos }\end{array}$ & $\begin{array}{l}\text { Diflubenzuron } \\
\text { Permethrin }\end{array}$ \\
& Permethrin & Temephos \\
Moderate $\left(5 \mathrm{ppm}<\mathrm{Z}_{\mathrm{IC} \mathrm{x}}<50 \mathrm{ppm}\right)$ & Alachlor & Propoxur \\
& Propoxur & Alachlor \\
& Atrazine & Atrazine \\
& Malathion & Carbaryl \\
& Methoprene & DDT \\
High $\left(\mathrm{Z}_{\mathrm{IC} \mathrm{x}} \leqslant 5 \mathrm{ppm}\right)$ & Carbaryl & Malathion \\
& DDT & Fenthion \\
& Fenthion & Toxaphene \\
& Toxaphene & Chlorpyrifos \\
& Gamma-BHC & Gamma-BHC \\
& Captan & Captan \\
& Chlorpyrifos & \\
& &
\end{tabular}

"Pesticides are ranked from the least toxic to the most toxic between and within each category based on their $\mathrm{Z}_{\mathrm{IC}}$ value (the concentration of each pesticide determined by its dose-mycelial inhibition line in which each isolate of $L$. giganteum produced viable zoospores).

zoosporogenesis in the $\mathrm{NC}$ isolate was less tolerant to these compounds than was mycelial growth. Extensive mycelial plasmolysis was observed in the $\mathrm{NC}$ isolate at its DDT $\mathrm{IC}_{10}(38.7 \mathrm{ppm}), \mathrm{IC}_{5}(12.1 \mathrm{ppm})$, and $\mathrm{IC}_{4}(6.2$ ppm), and gamma-BHC $\mathrm{IC}_{10}(1.0 \mathrm{ppm})$. Mycelial plasmolysis was not observed in the $\mathrm{NC}$ isolate in distilled water containing gamma-BHC at $0.8 \mathrm{ppm}\left(=\mathrm{IC}_{5}\right)$, or toxaphene at $6.1 \mathrm{ppm}\left(=\mathrm{IC}_{10}\right)$, but the fungus failed to produce discharge tubes. Discharge tubes were formed by the $\mathrm{NC}$ isolate is distilled water containing 2.8 (= $\left.\mathrm{IC}_{5}\right)$ and $2.0 \mathrm{ppm}\left(=\mathrm{IC}_{4}\right)$ of toxaphene, but the tubes were twisted and no vesicles or zoospores were produced. The NC isolate produced viable zoospores in a concentration equivalent to $1 \%$ inhibition of its mycelial growth rate on agar for each organochlorine insecticide.

Of the carbamate insecticides tested, carbaryl was more toxic than propoxur to the mycelial growth and zoospore production of both isolates. The LA isolate was more tolerant of carbaryl and propoxur than was the $\mathrm{NC}$ isolate, since the $\mathrm{NC}$ isolate did not produce discharge tubes, vesicles, and zoospores in distilled water containing lower concentrations of these insecticides than those in which the LA isolate produced zoospores.

The synthetic pyrethroid insecticide permethrin produced relatively high mycelial $\mathrm{IC}_{50}$ and $\mathrm{IC}_{10}$ values for both isolates. Extensive mycelial plasmolysis of the $\mathrm{NC}$ isolate was observed, however, in distilled water containing $175.5 \mathrm{ppm}\left(=\mathrm{IC}_{\mathrm{I0}}\right)$ of permethrin. At its mycelial $\mathrm{IC}_{5}(114.7 \mathrm{ppm})$ of permethrin, the $\mathrm{NC}$ isolate produced only twisted discharge tubes and, thus, no vesicles or zoospores. The LA isolate also produced only twisted discharge tubes at its mycelial $\mathrm{IC}_{10}$ of permethrin. The LA isolate was more tolerant of permethrin than was the NC isolate, since the former successfully produced viable zoospores in distilled water containing 88.6 ppm of permethrin, whereas the $\mathrm{NC}$ isolate failed to produce vesicles and zoospores at that concentration.

The insect growth regulator insecticide diflubenzuron caused no inhibition of mycelial growth rate of either isolate when incorporated into nutrient agar at concentrations up to and including $2,000 \mathrm{ppm}$. In addition, when tested for its effects on zoosporogenesis at 100 ppm, diflubenzuron did not inhibit the production of viable zoospores in either isolate. Although the other insect growth regulator insecticide, methoprene, was relatively innocuous to mycelial growth, it adversely affected zoospore production in both isolates at very low concentrations equivalent to less than the $\mathrm{IC}_{1}$ for mycelial inhibition. In distilled water containing methoprene concentrations which inhibited zoosporogenesis, extensive mycelial plasmolysis was also observed. The LA isolate was more tolerant of methoprene than was the $\mathrm{NC}$ isolate, since the LA isolate produced zoospores in distilled water containing $23.5 \mathrm{ppm}$ of methoprene, whereas the NC isolate did not.

There was little difference in tolerance between the two isolates to the herbicide alachlor, because their respective mycelial and zoospore responses were similar. The LA isolate was more tolerant than the $\mathrm{NC}$ isolate of atrazine, however, since the LA isolate had a higher mycelial tolerance. Of the two herbicides tested, atrazine was more toxic than alachlor to both isolates at the $10 \%$ level of mycelial inhibition.

The fungicide captan was very toxic to both isolates of $L$. giganteum. Although the mycelial growth rates of the NC and LA isolates on agar were inhibited by only $10 \%$ at 16.6 and $7.6 \mathrm{ppm}$ of captan, respectively, when the fungus was immersed in distilled water containing these and most lower concentrations of captan, extensive mycelial plasmolysis was observed. The mycelial growth of the NC isolate on agar was more tolerant of captan than was that of the LA isolate, as indicated by the higher $\mathrm{IC}_{50}$ and $\mathrm{IC}_{10}$ values of the $\mathrm{NC}$ isolate. Zoosporogenesis in both isolates appeared to be equally sensitive to captan, however, since both isolates produced viable zoospores in distilled water containing $0.3 \mathrm{ppm}$ of captan.

The ranking of the pesticides by toxicity is shown in Table 2. The pesticides were ranked from the least toxic to the most toxic between and within each arbitrary category of toxicity based on their $\mathrm{Z}_{\mathrm{ICx}}$ value; where $\mathrm{Z}$ is the concentration in $\mathrm{ppm}$ of each pesticide, determined by the percentage of mycelial growth rate inhibition $\left(\mathrm{IC}_{\mathrm{x}}\right)$ from each dose-mycelial inhibition line, in which each isolate of $L$. giganteum produced viable zoospores. The most toxic pesticides to both isolates of $L$. giganteum were: the fungicide captan; two of the organophosphate insecticides, chlorpyrifos and fenthion; and two of the organochlorine insecticides, gamma-BHC and toxaphene. Although the relative ranking of the pesticides within the "high-toxicity" category differ between isolates, all of these pesticides produced at least $10 \%$ inhibition of mycelial growth rate or inhibited zoospore production at concentrations below 5 ppm.

The pesticides ranked as "moderately toxic" to $L$. giganteum were those which resulted in a $10 \%$ inhibition 
of mycelial growth rate or inhibited zoospore production at concentrations between 5 and $50 \mathrm{ppm}$. Moderately toxic pesticides to both isolates of $L$. giganteum were: the organophosphate insecticide malathion; the carbamate insecticide carbaryl; the herbicides alachlor and atrazine; and the insect growth regulator insecticide methoprene. In addition, the carbamate insecticide propoxur was moderately toxic to the $\mathrm{NC}$ isolate, whereas the organochlorine insecticide DDT was moderately toxic to the LA isolate.

The pesticides ranked in the "low-toxicity" category were those which caused a $10 \%$ inhibition of mycelial growth rate and failed to inhibit zoospore production at some concentration greater than $50 \mathrm{ppm}$. The insect growth regulator insecticide diflubenzuron was the least toxic pesticide to both isolates of $L$. giganteum. In addition, the synthetic pyrethroid insecticide permethrin and the organophosphate insecticide temephos showed low toxicity to both isolates. Propoxur also showed low toxicity to the LA isolate.

The various groups of pesticides showed certain trends which transcend the arbitrary categories in which they were ranked. First, the fungicide captan and the organochlorine insecticides were the most toxic pesticides to both isolates. The organophosphate insecticides, with the exception of temephos, were also quite toxic. The herbicides and carbamate insecticides showed moderate toxicity, whereas the insect growth regulator insecticides (especially diflubenzuron), the synthetic pyrethroid permethrin, and the organophosphate insecticide temephos were the least toxic and thus most compatible of the pesticides tested to both isolates of $L$. giganteum.

\section{Discussion}

L. giganteum exhibited several types of responses upon exposure to various concentrations of pesticides in distilled water. Mycelial plasmolysis was often observed in distilled water containing relatively high pesticide concentrations. A second toxic response was observed when a pesticide inhibited the formation of discharge tubes (e.g., $\mathrm{NC}$ isolate in $0.8 \mathrm{ppm}$ of gamma-BHC). A third toxic response was when the fungus produced normal or sometimes twisted discharge tubes but failed to produce vesicles and, subsequently, zoospores (e.g., NC isolate in $2.0 \mathrm{ppm}$ of toxaphene). A fourth type of response was observed when the fungus was exposed to pesticide concentrations which did not cause mycelial plasmolysis nor inhibit discharge tube and vesicle formation. In this case, the fungus produced viable zoospores, some of which encysted and germinated on the WHS agar sections in the distilled-water-pesticide solutions.

There are several factors which may affect the responses of $L$. giganteum to the various pesticides included in this study. First, the physical nature of the culture medium may affect the fungitoxicity of certain pesticides. For example, mycelial plasmolysis in $L$. giganteum was observed in water containing either chlorpyrifos or DDT concentrations far below their respective mycelial $\mathrm{IC}_{50}$ values in agar. Ramaraje Urs et al. (1967) reported a similar response in Beauveria bassiana Bals.
Vuillemin. They found that mycelial growth was completely inhibited in liquid medium containing $0.1 \%$ DDT, yet the fungus grew normally on nutrient agar containing the same concentration of DDT. Thus, the $\mathrm{IC}_{\left.5_{0}\right)}$ values for mycelial growth rate on agar may underestimate the toxicity of certain pesticides, especially those in which mycelial plasmolysis was observed in water containing lower pesticide concentrations. Second, the nutritional composition of the environment in which $L$. giganteum is exposed may affect its mycelial growth rate. Dalton and Smith (1971) reported that $25 \mathrm{ppm}$ of DDT had no effect on the mycelial growth of the aquatic fungus $\mathrm{Hel}$ iscus submersus when the insecticide was incorporated into culture media containing either glucose or fructose. However, when 25 ppm DDT was incorporated into culture media containing either maltose, sucrose, or lactose as a carbohydrate source, mycelial growth was retarded. Another factor which may affect the toxicity of certain pesticides to $L$. giganteum is the possible interaction between the fungus, the pesticide, and the organic solvent used to dissolve the pesticide. Burrell and Corke (1980) showed that additive, antagonistic, and synergistic interactions can occur between certain phytopathogenic fungi, and acetone-benomyl or acetone-captan mixtures in in vitro tests. Although our study did not examine the possible interactions between $L$. giganteum and various acetone-pesticide combinations, an attempt was made to minimize interactions by using acetone concentrations which had a negligible effect on mycelial growth and zoosporogenesis. In any event, since the data in this study were gathered from laboratory experiments on artificial media, the results should be considered as estimates of the toxicity of pesticides to $L$. giganteum until final tests are conducted under field conditions, as advocated by Jacques and Morris (1981) for all entomopathogens and chemical pesticides.

Zoospore production in both isolates of L. giganteum was less tolerant than mycelial growth to most pesticides, since zoospore production was often completely inhibited in lower pesticide concentrations than those which partially inhibited mycelial growth on agar, or resulted in mycelial plasmolysis in water. Merriam and Axtell (1982) found that zoosporogenesis in L. giganteum was ca. 22 times more sensitive to $\mathrm{NaCl}$ than was mycelial growth.

There were inter-isolate differences in the degree to which zoosporogenesis was less tolerant than mycelial growth to pesticides, and these differences were evident for most of the pesticides tested. For instance, the difference in fenthion tolerance between zoosporogenesis and mycelial growth of the LA isolate was one-tenth that of the $\mathrm{NC}$ isolate, since the LA isolate produced zoospores in water containing a concentration of fenthion equal to it mycelial $\mathrm{IC}_{10}$, whereas the $\mathrm{NC}$ isolate only produced zoospores in water containing a fenthion concentration equal to its mycelial $\mathrm{IC}_{1}$ (Table 1).

The results of this study show that the NC and LA isolates differ in their tolerance toward certain pesticides. The $\mathrm{NC}$ isolate was more tolerant of malathion than was the LA isolate. The LA isolate, however, was more tolerant of chlorpyrifos, fenthion, DDT, toxa- 
phene, carbaryl, propoxur, permethrin, and methoprene than was the NC isolate. These inter-isolate differences in pesticide tolerance may be important considerations in the potential use of either isolate as a microbial control agent for mosquitoes in pesticide contaminated habitats. Olmert and Kenneth (1974) also found differences in tolerance to certain insecticides and fungicides among isolates of the entomophathogenic fungi, Verticillium spp. and B. bassiana.

Certain pesticides were very toxic to both isolates of L. giganteum. Captan was one of the most toxic pesticides tested, and since it is a fungicide, its high toxicity to L. giganteum is not surprising. The fungitoxicity of captan is attributed to the moiety $-\mathrm{SSCl}_{3}$, a group found in many fungicides. The organochlorine insecticides gamma-BHC, toxaphene, and DDT, and the organophosphate insecticides chlorphyrifos and fenthion, were also very toxic to $L$. giganteum. With the exception of fenthion, all of these insecticides are chlorinated compounds. Many fungicides are chlorine-substituted aromatic compounds, such as hexachlorobenzene. Thus, the high toxicity of gamma-BHC and L. giganteum may be related to the chlorine constituents of the compound. The high fungus toxicity of fenthion and DDT may be related to their respective $-\mathrm{SCH}_{3}$ and $-\mathrm{CCl}_{3}$ moieties, which are both similar to the fungitoxic moiety $-\mathrm{SCCl}_{3}$ of captan. In addition, DDT contains chlorine-substituted aromatic groups. Other researchers have reported that certain insecticides adversely affect other fungal pathogens of mosquitoes. Ramaraje Urs et al. (1967) found that gamma-BHC completely inhibited the mycelial growth of B. bassiana and Metarrhizium anisopliae Metch. Sorokin when incorporated into nutrient agar at concentrations of $400 \mathrm{ppm}$ and above. In addition, their data showed both 400 ppm of DDT and 400 ppm of malathion inhibited the mycelial growth of $B$. bassiana by 37.1 and $57.1 \%$, respectively. At the same concentration $(400 \mathrm{ppm})$, DDT and malathion inhibited the mycelial growth of $M$. anisopliae on agar by 54.3 and $60.0 \%$, respectively. Olmert and Kenneth (1974) reported that carbaryl caused slight inhibition at 2,000 ppm, but that 4,000 ppm of chlorpyrifos did not inhibit the mycelial growth of $B$. bassiana on nutrient agar.

The integrated pest management approach to mosquito control includes the use of chemical, biological, and cultural and physical methods of mosquito control (Axtell 1979). In this context, it is conceivable that as a potential microbial control agent for mosquitoes, $L$. giganteum may be exposed to or used together with certain mosquito larvicides. The results of this study show that the NC and LA isolates of L. giganteum were compatible with the following mosquito larvicides at their recommended rates of application (as calculated in ppm on the basis of the weight of active ingredients per acre-ft of water; see Rathburn [1979]): methoprene (0.010 ppm), fenthion $(0.037 \mathrm{ppm})$, malathion $(0.185 \mathrm{ppm})$, and temephos $(0.185 \mathrm{ppm})$. Both isolates of $L$. giganteum produced viable zoospores in water and grew vegetatively on agar containing higher concentrations of these insecticides (Table 1) than those which are recommended for field use. In the case of chlorpyrifos, how- ever, the NC isolate of L. giganteum was inhibited at $1.4 \mathrm{ppm}$, a concentration below the recommended rate of chlorpyrifos application (1.5 ppm), and thus appears not to be compatible with this insecticide. Although the LA isolate was more tolerant of chlorpyrifos than was the NC isolate, the compatibility of the LA isolate with chlorpyrifos at $1.5 \mathrm{ppm}$ is uncertain, since it produced zoospores at $1.4 \mathrm{ppm}$, but failed to produce zoospores at $3.5 \mathrm{ppm}$.

L. giganteum may be exposed to insecticides (other than mosquito larvicides), herbicides, and fungicides from agricultural uses through the transport and accumulation of these pesticides in bodies of water where the fungus is already established. Since several factors may affect the amounts of pesticides which reach aquatic systems via runoff, it is difficult to predict the amount of pesticide runoff and, hence, the biological significance of these concentrations. These factors include: the time interval between application of the pesticide and first rainfall, and the frequency, intensity and duration of rainfall (White et al. 1967); and the rate of pesticide application, distance between treated areas and bodies of water, and the slope and cover of treated land (Trichell et al. 1968). Physical and chemical properties of pesticides can also affect the loss of pesticides into surface water. Although they are generally insoluble and not readily degraded in water, organochlorine compounds such as DDT and toxaphene are absorbed to sediment and mainly transported in this state (Bradley et al. 1972). Skaggs et al. (1980) detected alachlor residues in drainage water as high as $79 \mathrm{ppb}$ after careful herbicide application, whereas alachlor residues as high as $2.6 \mathrm{ppm}$ were detected in drainage water when probably contaminated by either direct application or heavy drift of herbicide spray. Although toxic pesticides which readily degrade may thus become less hazardous than more persistent pesticides to aquatic organisms, under certain circumstances (e.g. substantial rainfall and pesticide runoff immediately after application, or pesticide drift during application into bodies of water) when significant degradation of nonpersistent pesticides has not occurred, residues from these pesticides may be sufficient enought to be toxic to $L$. giganteum.

In situations where $L$. giganteum may be introduced into bodies of water already contaminated by pesticides, or which traditionally receive pesticide input, it is important to know the identity and concentration of the various pesticides to which the fungus will be exposed. By conducting an analytical pesticide residue analysis and evaluating this residue data with respect to the toxicity of various pesticides to different isolates of $L$. giganteum, one can decide if the use of either isolate of L. giganteum as a microbial control agent for mosquitoes in pesticide-contaminated sites would be appropriate in respect to the efficacy and persistence of the fungus.

\section{Acknowledgment}

We thank Nolan Newton of this department for his assistance in laboratory work and statistical analysis. This research was supported in part by NIH grant IROI AI 17024. 


\section{REFERENCES CITED}

Axtell, R. C. 1979. Principles of integrated pest management (IPM) in relation to mosquito control. Mosq. News 39: 709-718.

Barr, A. J., J. H. Goodnight, J. P. Sall, W. H. Blair, and D. M. Chilko. 1979. SAS user's guide. SAS Institute INC, Raleigh, N.C. 494 pp.

Bradley, J. R., Jr., T. J. Sheets, and M. D. Jackson. 1972. DDT and toxaphene movement in surface water from cotton plots. J. Environ. Qual. 1: 102-105.

Burrell, R. E., and C. T. Corke. 1980. Interactions of the solvent acetone with the fungicides benomyl and captan in fungal assays. Bull. Environ. Contam. Toxicol. 25: 554 561 .

Couch, J. N. 1935. A new saprophytic species of Lagenidium, with notes on other forms. Mycologia 27: 376-387.

Dalton, S. A., and K. A. Smith. 1971. DDT and river fungi. Proc. 1st Int. Mycol. Congr. Trans. Br. Mycol. Soc. 58: Suppl. 28.

Federici, B. A. 1981. Mosquito control by the fungi Culicinomyces, Lagenidium and Coelomomyces. In H. D. Burges, [ed.], "Microbial control fo pests and plant diseases 197080. Academic Press, Inc., New York.

Glenn, F. E., and H. C. Chapman. 1978. A natural epizootic of the aquatic fungus Lagenidium giganteum in the mosquito Culex territans. Mosq. News 38: 522-524.

Jacques, R. P., and O. N. Morris. 1981. Compatibility of pathogens with other methods of pest control and with different crops. In H. D. Burges, [ed.], Microbial control of pests and plant diseases 1970-1980. Academic Press, Inc., New York.

Jaronski, S. T., and R. C. Axtell. 1982. Effects of organic water pollution on the infectivity of the fungus Lagenidium giganteum (Oomycetes: Lagenidiales) for mosquito larvae (Culex quinquefasciatus): field and laboratory evaluation. J. Med. Entomol. 9: 255-262.
McCray, E. M., Jr., C. J . Umphlett, and R. W. Fay. 1973a. Laboratory studies on a new fungal pathogen of mosquitoes. Mosq. News 33: 54-60.

McCray, E. M., Jr., D. J. Womeldorf, R. C. Husbands, and D. A. Eliason. 1973b. Laboratory observations and field tests with Lagenidium against California mosquitoes. Proc. Calif. Mosq. Control Assoc. 41: 123-128.

Merriam, T. L., and R. C. Axtell. 1982. Salinity tolerance of two isolates of Lagenidium giganteum (Oomycetes: Lagenidiales), a fungal pathogen of mosquito larvae. J. Med. Entomol. 19: 388-393.

Olmert, I., and R. G. Kenneth. 1974. Sensitivity of the entomopathogenic fungi, Beauvereia bassiana, Verticillium lecanii, and Verticillium $\mathrm{sp}$. to fungicides and insecticides. Environ. Entomol. 3: 33-38.

Ramaraje Urs, N. V., H. C. Govindu, and K. S. Shivashandara Shastry. 1967. The effect of certain insecticides on the entomogenous fungi Beauveria bassiana and Metarrhizium anisopliae. J. Invertebr. Pathol. 9: 398-403.

Rathburn, C. B., Jr. 1979. Insecticides for the control of mosquitoes and other Diptera. Mosq. News 39: 58-63.

Roberts, D. W. and A. S. Campbell. 1977. Stability of entomopathogenic fungi. Misc. Publ. Entomol. Soc. Am. 10: 19-76,

Skaggs, R. W., J. W. Gilliam, T. J. Sheets, and J. S. Barnes. 1980. Effect of agricultural land development on drainage waters in the North Carolina tidewater region. Water Resources Research Institute of the University of North Carolina. Rep. No. 159.

Trichell, D. W., H. L. Morton, and M. G. Merkle. 1968. Loss of herbicides in runoff water. Weed Sci. 19: 447-449.

Umphlett, C. J., and C. S. Huang. 1970. Lagenidium culicidum as an agent of biological control of mosquitoes. Bull. Assoc. Southeast. Biol. 17: 68 .

White, A. W., A. P. Barnett, B. G. Wright, and J. H. Holladay. 1967. Atrazine losses from fallow land caused by runoff and erosion. Environ. Sci. Technol. I: 740-744. 